\title{
Multicentric Castleman's Disease with Disseminated Kaposi's Sarcoma: A Report of Prolonged Misdiagnosis
}

\author{
Xiao $\mathrm{Hu}^{1}$, Monika Pilichowska ${ }^{2}$ and Cindy Varga ${ }^{3 *}$ \\ ${ }^{1}$ Department of Medicine, Tufts Medical Center, USA \\ ${ }^{2}$ Department of Pathology and Laboratory Medicine, Tufts Medical Center, USA \\ ${ }^{3}$ Division of Hematology, Department of Medicine, The John C Davis Myeloma and Amyloid Program, Tufts Medical \\ Center, USA
}

*Corresponding author: Cindy Varga, MD, Division of Hematology, Department of Medicine, The John C Davis Myeloma and Amyloid Program, Tufts Medical Center, 800 Washington St, Boston, MA 02111, USA, Tel: 617-636-6227, Fax: 617$636-8538$

\section{Dear Editor,}

Castleman's Disease is a rare and benign lymphoproliferative disease. Human immunodeficiency virus (HIV) and human herpesvirus 8 (HHV-8) infections are highly associated with multicentric Castleman's disease (MCD) [1]. Studies suggest HHV-8 plays an important role in the pathogenesis by encoding a viral form of IL-6 [2], and MCD tends to be more common in the highly active antiretroviral therapy (HAART) era [3]. There is no standard of treatment for MCD. Many single or combined cytotoxic drugs have been reported to provide durable remissions $[4,5]$. Rituximab remains mainstay treatment because of its substantial safety, efficacy and survival benefit [6]. Concurrent use of liposomal doxubicin is suggested as rituximab can promote the progression of Kaposi's sarcoma (KS) [7]. The prognosis of untreated disease is poor and thus, early recognition and timely initiation of therapy are critical. Here we report a case of MCD and disseminated Kaposi's sarcoma that went unrecognized leading to unnecessary delays in initiating treatment.

A 54-year-old gentleman was diagnosed with acquired immunodeficiency syndrome (AIDS) when presenting with shingles in 2016. He was started on HAART but soon noted a very rapid increase in the size of his cervical, axillary and inguinal lymph nodes, along with profound constitutional symptoms.
Immune reconstitution syndrome was suspected and prednisone was tried but did not alleviate symptoms. One month later, a core biopsy of the left axillary lymph node was undertaken and demonstrated predominantly mature kappa-light chain restricted plasma cells (Figure 1). Flow cytometry was negative for a clonal population. One month later, the patient was referred to the Haematology department for a plasma cell neoplasm. Initial blood work did not reveal

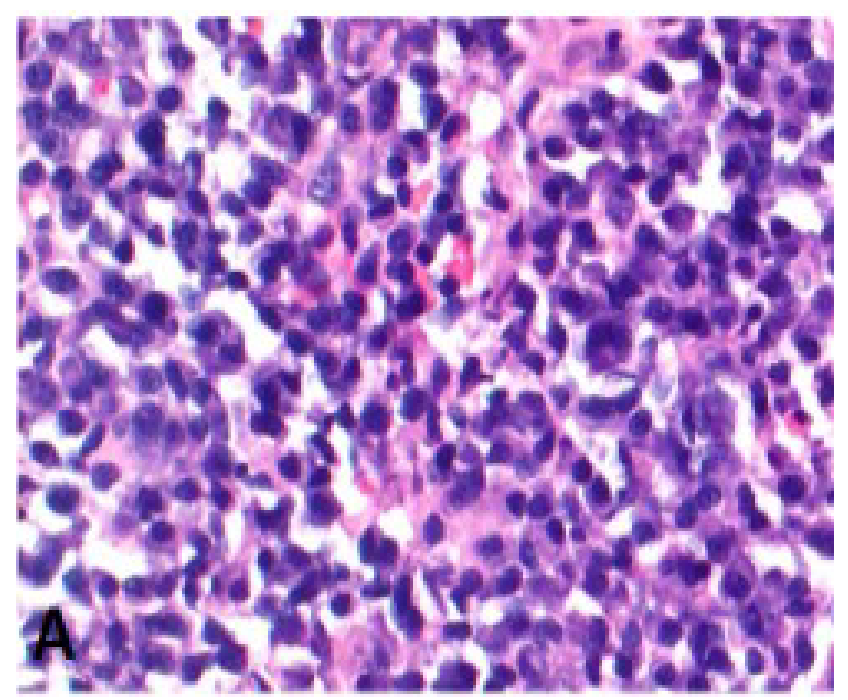

Figure 1: Core biopsy of the left axillary lymph node Sheets of plasma cells.

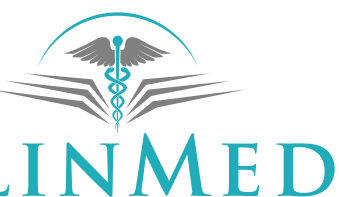

INTERNATIONAL LIBRARY
Citation: Hu X, Pilichowska M, Varga C (2020) Multicentric Castleman's Disease with Disseminated Kaposi's Sarcoma: A Report of Prolonged Misdiagnosis. Int J Blood Res Disord 7:051. doi. org/10.23937/2469-5696/1410051

Accepted: June 04, 2020: Published: June 06, 2020

Copyright: (C) $2020 \mathrm{HuX}$, et al. This is an open-access article distributed under the terms of the Creative Commons Attribution License, which permits unrestricted use, distribution, and reproduction in any medium, provided the original author and source are credited. 


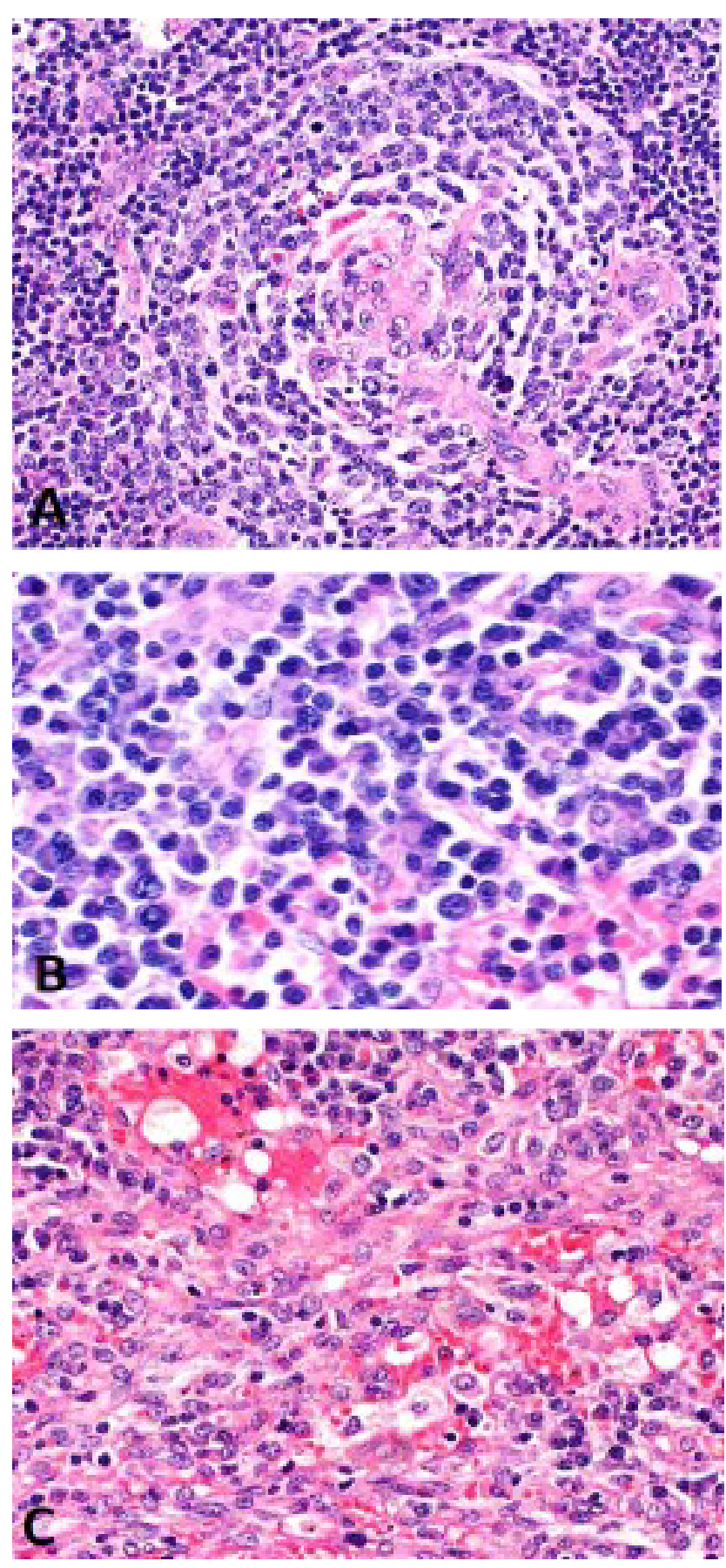

Figure 2: Excisional biopsy of a left cervical lymph node A) Lymph node showing Castlemen features; B) Plasma cell rich areas; C) Area involved by Kaposi's sarcoma.

a monoclonal gammopathy. A bone marrow biopsy was negative for a hematologic malignancy. Computed tomography (CT) chest/abdomen/pelvis and positron emission tomography (PET) were notable for diffuse lymphadenopathy above and below the diaphragm with splenomegaly. An excisional biopsy of a left cervical node revealed nodal involvement by $M C D$ with metastatic $K S$ (Figure $2 \mathrm{~A}$, Figure $2 \mathrm{~B}$ and Figure $2 \mathrm{C}$ ). Blood work confirmed an HHV-8 level of $>10,000,000$ copies $/ \mathrm{mL}$.

The patient was treated with liposomal doxorubin and rituximab without a clinical response. Etoposide was added but without effect. Therapy was upgraded to cyclophosphamide, adriamycin, vincristine, and prednisone (CHOP) with immediate resolution of symptoms. After completion of 6 planned cycles, a PET-CT confirmed a complete response and a repeat HHV-8 level was $<1000$ copies $/ \mathrm{mL}$. Unfortunately, the patient then reported a rapidly progressive painful erythematous rash in both lower extremities. After several weeks of failing antibiotic therapy, a punch biopsy of the skin demonstrated disseminated $K S$ and a repeat HHV-8 level was at $>14,000$ copies $/ \mathrm{mL}$. Vinblastine was initiated with drastic improvement in his symptoms.

Castleman's disease is a great mimicker of multiple illnesses due to its various manifestations. In this case, the plasma cells on the core biopsy misled providers to a diagnosis of myeloma. An initial excisional biopsy with careful pathologic review is required to properly capture the nodal architecture. This delay led to the aggressive widespread dissemination of the disease requiring a 5-drug regimen which is an uncommon approach in this disease. The disseminated KS, presenting as rapid painful rashes instead of singular purple skin lesions, also deceived the medical team. This case illuminates the diagnostic complexity of lymphadenopathy workup in HIV+ patients and highlights the importance of pursuing ample tissue in order to make an accurate and timely diagnosis.

\section{Acknowledgments}

We would like to acknowledge this patient for sharing the story.

\section{Compliance with Ethical Standards}

\section{Funding}

This study was not funded.

\section{Conflict of Interest}

Drs. Hu, Varga and Pilichowska have no conflicts of interest to declare.

\section{Ethical approval}

This article does not contain any studies with human participants performed by any of the authors.

\section{References}

1. Soulier J, Grollet L, Oksenhendler E, P Cacoub, D Cazals-Hatem, et al. (1995) Kaposi's sarcoma-associated herpesvirus-like DNA sequences in multicentric Castleman's disease. Blood 86: 1276-1280.

2. Polizzotto MN, Uldrick TS, Wang V, Karen Aleman, Kathleen M Wyvill, et al. (2013) Human and viral interleukin-6 and other cytokines in Kaposi sarcoma herpesvirus-associated multicentric Castleman disease. Blood 122: 41894198.

3. Mylona EE, Baraboutis IG, Lekakis LJ, Georgiou O, Papastamopoulos V, et al. (2008) Multicentric castleman's disease in HIV infection: A systematic review of the literature. AIDS Rev 10: 25-35.

4. El-Osta HE, Kurzrock R (2011) Castleman's disease: From 
basic mechanisms to molecular therapeutics. Oncologist 16: 497-511.

5. Seo HY, Kim EB, Kim JW, Shin BK, Kim SJ, et al. (2009) Complete remission in a patient with human herpes virus-8 negative multicentric Castleman disease using $\mathrm{CHOP}$ chemotherapy. Cancer Res Treat 41: 104-107.

6. Gerard L, Berezne A, Galicier L, Meignin, Martine Obadia, et al. (2007) Prospective study of rituximab in chemotherapy-dependent human immunodeficiency virus associated multicentric Castleman's disease: ANRS 117 CastlemaB Trial. J Clin Oncol 25: 3350-3356.

7. Uldrick TS, Polizzotto MN, Aleman K, Kathleen M Wyvill, Vickie Marshall, et al. (2014) Rituximab plus liposomal doxorubicin in HIV-infected patients with KSHV-associated multicentric Castleman disease. Blood 124: 3544-3552. 